\title{
AAPS Workshop Report on Biorelevant In Vitro Performance Testing of Orally Administered Dosage Forms
}

\author{
Bethesda, MD, March 18-19, 2013 \\ Summarized on behalf of the Programming Committee by Johannes Krämer
}

S uccessful authorization of drugs requires mostly the proof of bioavailability requiring numerous studies in humans. Complementation of clinical testing by meaningful in vitro laboratory methods would speed up authorization procedures. An increasing number of poorly water-soluble drug substances used for oral administration and the limitations of the traditional procedures require ongoing research and development to link in vitro and in vivo performance testing. More than 80 scientists, mainly from the United States, Europe, and Japan, discussed the progress in this field starting with the human physiology and in vitro release testing to linking the two aspects by establishing relations or correlations between in vitro and in vivo. Regulatory authorities participated with the goal of integrating novel procedures in their decision-making process, not only to benefit patients but also to limit the burden on industry.

\section{NOYES-WHITNEY EQUATION OUTDATED?}

This very challenging question was asked by James Brasseur, Penn State University, when comparing the hydrodynamics in the classical paddle instrument with those in the human intestine after food intake. According to his investigations, the differences between the geometries of the in vivo and the in vitro sites of dissolution are not critical. Other aspects, such as the confinement in the human intestine, are of greater relevance. In the human GI tract, two motility patterns prevail, the "propagation" caused by peristalsis with a more circular pattern and the "standing" due to segmentation characterized by collapsing and reopening movements. In the human gut, dissolution of solids is mostly diffusion dominated and driven by the relationship of particle size to diffusion layer thickness, expressed as the so-called Sherwood number. He pointed out that classical models assuming a constant diffusion layer thickness, such as the Noyes-Whitney equation first published in the 19th century, are outdated. Instead, his quasi-steady-state model shows that differences in the dissolution rate in a diffusion-controlled dissolution environment are a result of differences in the degree of confinement. Its importance increases with particle size and in those regions of the Gl lumen where segments build confinements, such as in the small intestine.

\section{BIORELEVANT MEDIA AND BEYOND}

The term biorelevant used by most scientists in the area of dissolution still seems to need ongoing discus- sion for a precise definition. The use of biorelevant media is increasing also because they are now available in a powder version ready for reconstitution. To understand their benefit, knowledge of human physiology is needed. Based upon the hypothesis that the closer the test conditions are to the Gl physiology, the better the chances of predicting product performance, Dressman showed the relevant differences in the composition of fluids at fasted and fed states. Besides $\mathrm{pH}$, osmolarity, buffer capacity, and the activity of enzymes at the sites of dissolution and absorption, the mechanical effect in different parts of the Gl tract is largely different. Moreover, gastric emptying is dependent on volume, temperature, and calorific content of nutrients concomitantly taken with the solid oral dosage forms. Once particles are emptied from the stomach, the transit throughout the small intestine is rather constant and takes three to five hours on the average. As the most important absorption time is in the small intestine, this residence time is more important for the amount absorbed than the dissolution rate. The central statement of Dressman is that for poorly soluble drug candidates, biorelevant in vitro performance tests are required to forecast in vivo product performance.

With QbD in mind, successful dissolution method development should follow a mechanistic approach rather than rely on empirical processes is the strong opinion of Christos Reppas from University of Athens. Together with Jennifer Dressman, Gordon Amidon, and Vinod Shah (1), he is the coauthor of one early pivotal paper on biorelevant media published. Fifteen years later, he extended the term biorelevant to less complex media such as buffer solutions because in vivo dissolution may or may not be the rate-limiting step compared with absorption. He pointed out that simple buffer systems simulating the luminal $\mathrm{pH}$ can be biorelevant for IR products of BCS Class 1 and Class 3 APIs. However, a more detailed simulation of luminal composition may be needed for a medium to be biorelevant in cases where API-drug product performance becomes very sensitive to luminal composition. Based on his research using human gastrointestinal fluid aspirates, he suggests performing experiments with biorelevant media for a better understanding of in vivo drug behavior. For lipophilic APIs and PBPK modeling, the benefits are an estimate of luminal solubility and hence luminal dissolution kinetics as well as stability in the $\mathrm{Gl}$ tract including physical stability phenomena such as precipitation. 


\section{MORE SOPHISTICATED DISSOLUTION APPARATUS TO COME?}

Precipitation of a drug substance after dissolution may occur along the GI tract. To explain the results of bioavailability studies, a posteriori mixed dissolutionprecipitation models are used in industry. The so-called mixed-tank models allow a defined transfer of drug in solution from a dissolution tank into a precipitation tank containing different media. The combination of the dissolution process with an appropriate rate of transfer from one compartment to another allows modeling of in vivo findings. This work goes back to the scientific work of mainly Edmund Kostewicz and Lyn Hughes. Techniques of this kind are used in industry as reported by David Sperry of Eli Lilly and James Butler of Glaxo Smith Kline. At Lilly, the combined dissolution and precipitation model is integrated in the method development process following a clearly defined decision tree. In development, it is used to:

- Estimate in vivo solubility.

- Estimate drug substance dispersability.

- Determine the propensity for drug substance precipitation.

- Optimize formulations.

- Design QC test conditions.

- Assess the risk of changes observed with QC test conditions.

- Assess bioavailability risk.

- Input dissolution rates for absorption modeling.

As one consequence, it allows the separation of dosage form-related phenomena leading to certain invasion kinetics from the physiology-governed transfer of solutions to absorption sites. Butler strongly emphasized the routine use of precipitation models. He reported on the Innovative Medicines Initiative (IMI) project called OrBiTo, which stands for Oral Biopharmaceutics Tools. Under the leadership of Bertil Abrahamsson, OrBiTo bundles the activities of 12 pharmaceutical companies, 11 universities, and other participants. The goal is to enhance the knowledge of how orally administered drugs are taken up from the $\mathrm{Gl}$ tract into the body. This knowledge aims at creating new lab tests and computer models that will better predict the performance of these drugs in patients. Butler (2) also described his developability classification system as an application of biopharmaceutics concepts to formulation development. It includes a subcategorization of BCS Class 2 and Class 4 with regard to potential supersaturation phenomena in the Gl tract.

From his more than 25 years in R\&D at Astra Zeneca, Abrahamsson advertised the use of in vitro performance testing: "In vitro dissolution is the most important test in development of ER dosage forms...." He also warned "... but it is a waste of time if not in vivo predictive!" From his daily experience and challenges, he drew a line from in vitro testing to in vivo performance for a product and the claim of developing formulations for which drug dissolution is unaffected by physiological conditions. This clearly indicates that with a required dose and a given absorption capacity, in vivo performance largely depends on the sensitivity of a product to changes of the dissolution environment in the human $\mathrm{Gl}$ tract. The transit rate is considered the other variable for bioavailability in comparison with the built-in dissolution rate.

\section{CONVOLUTION OR DECONVOLUTION TO ESTABLISH AN IVIVR}

Once suitable in vitro dissolution data are obtained, one way of generating corresponding in vivo profiles is by computer simulation based on appropriate pharmacokinetic models. This convolution of plasma concentration-versus-time profiles is successfully used in industry for product development as Filippos Kesisoglou from Merck and Co. reported. One of the advantages of modeling is to provide results where IVIVC is difficult to obtain or not feasible at all. Modeling does not require clinical data on different formulations. It may include the quantitative relationship of dissolution and absorption in vivo even in cases where absorption is rate limiting. This is of great importance for IR products and best with linear pharmacokinetics. Modeling may even overcome the limitations of IVIVC, where correlations are limited to "similar" formulation changes. According to his presentation, proper use of modeling allows the simplification of dissolution models once the mechanistic details have been investigated by more detailed procedures, as described above. He described combining data of simpler dissolution tools rather than developing "complex" dissolution systems. As a consequence, biorelevant in vitro performance testing may be performed even with compendial instruments.

The classical approach to correlate in vitro with in vivo performance data is the IVIVC preferred by authorities and described in the recently revised USP General Chapter $<1088>$. The three-level correlation scale reaches from point-to-point correlation, called Levy plot, to levels after data reduction, where entire profiles are represented by single parameters such as $C_{\max }$ or MRT. As one common prerequisite, classical IVIVC is based on the assumption that in vivo dissolution is the rate-limiting step. This explains why IVIVCs are most successful with ER dosage forms and may fail with IR dosage forms. James Polli, University of Maryland, provided an algorithm to cover the cases where absorption is the rate-limiting step. His algorithm assumes that in vitro and in vivo dissolution rates are similar and is based on a one-compartment open model with first-order elimination-invasion kinetics. This model has the great benefit of explaining quantitatively incomplete absorption of the dose in the development of dosage forms at a given dissolution rate. The algorithm 
helps to establish a linear relationship as the ideal case but extends to nonlinear function plots for absorption-limited pharmacokinetic behavior of dosage forms.

\section{REGULATORY AUTHORITIES' CONTRIBUTION TO PROGRESS}

Despite of the advances in science, authorities tend to take a conservative approach. As Christine Moore from the United States FDA ONDQA pointed out, the body of knowledge does not yet suffice to recommend a biowaiver for BCS Class 2 drugs based on the use of biorelevant media. Otherwise, she sees the objectives of dissolution in formulation development and process development during development and in linking clinical batches in vitro performance to marketed batches as well as to prove lotto-lot consistency. In the case of a product where the in vitro performance is linked to its in vivo performance, the in vitro data may be used:

- To set specifications.

- As an integral part of QbD.

- To assess product variability.

- To waive BE studies.

There is no regulatory requirement for biorelevant in vitro testing according to Moore. She described the term "biorelevant" as an attribute for a laboratory (in vitro) test that utilizes conditions and environments mimicking in vivo environments and conditions, or a laboratory test capable of predicting in vivo response not necessarily mimicking in vivo environment but providing quantitative predictions.

Arzu Selen from ONDQA emphasized that the discrepancy between in vitro performance testing as a quality control tool and the attempt to link results to the in vivo performance of a drug may be bridged by QbD. She referred to the early work of Gerhard Levy in 1961, who established a relationship between differences in vitro performance of multisource aspirin brands and the incidence of their side effects. From differences between products or those detected in development, she extended the goal of in vitro performance testing to showing similarity (e.g., after changes). One of her central statements was that in vitro performance testing helps in the "learning" process about the properties of a particular product. Complex media such as FaSSiF or milk may help to broaden this learning effect.

Current FDA regulatory guidances include the biowaiver note for guidance, the guidance on development of ER dosage forms, as well as those covering dissolution testing, with some dating back to 1997. Barbara Davit from the FDA Office of Generic Drugs explained that the FDA is asking for biorelevant data to set specifications, which in an ideal case will accompany the product throughout its entire lifecycle. The specifications and the corresponding in vitro performance testing methods are closely linked to the product. OGD assumes that for IR products, generic dissolution methods may exist including those available in the USP but that ER products require individual method development. This development should aim at setting meaningful specifications based on the in vivo performance of the target lot. Meaningful specifications may also help to apply the biowaiver concept on different strengths of one product being compositionally proportional. According to Davit, dissolution data may also support the proof that a product is rugged. This concept is widely used for in vitro testing of dose-dumping caused by alcohol-containing beverages and is recommended for opioids.

EMA Guideline on Quality of Oral Modified Release Products is a pivotal document in the EU for the assessment of applications for marketing authorization for both new and established drugs. Henrike Potthast reported from her experience in the German regulatory authority (BfArM) and EMA. As in the United States, the BCS concept provides some framework for regulatory guidance and hence decision-making. In vitro dissolution testing provides information on the test batches used in bioavailability and bioequivalence studies and in pivotal clinical studies to support specifications for in vitro testing. With this in mind, in vitro dissolution testing is considered a tool in quality control to demonstrate manufacturing consistency of a product and to provide information on a reference product used in BA/BE studies as well as in pivotal clinical studies.

An observer at the AAPS workshop noticed the inconsistency between FDA and EU about whether an in vitro method developed for a given test product may be used by extension to characterize the reference product as well.

With ICH Q4B in place, the Japanese practice seems to differ slightly from that of the other ICH members. However, the main point of the harmonization is the equipment and interpretation. Dissolution test media and so forth have to be developed on a case-by-case basis for a given product as explained in ICH Q4B Annex No. 6. Chikako Yomota, Drug Division, National Institute of Health Sciences, Japan, gave an outline of how the Japanese guidelines (http://www.nihs.go.jp/drug/DrugDiv-E.html) apply at the regulatory authorities. She exemplified the use of surfactants for dissolution testing of poorly soluble drugs. In the past, the use of SDS was not encouraged in Japan. However, this has changed recently, and the use is now permitted in certain cases. Other than in the United States and Europe, solubility is not defined by the BCS but depends on the dissolution profiles of products. The way to characterize products follows the scheme of Jerome Skelly, proposed in 1987, which he called topographical characterization. It includes multiple dissolution tests under varying conditions such as $\mathrm{pH}$, agitation, and surfactant concentration. 


\section{PROGRESS AND COMPLETE INFORMATION: WHERE DO WE GO FROM HERE?}

In summary, combinations of complex dissolution media and apparatus are best suited for the determination of in vivo dissolution. However, if additional steps are involved between in vivo dissolution and the resulting effect on the bioavailability of a product (absorption and gastrointestinal transit), holistic approaches based on less complex media and apparatus may be preferred.

At the end of the workshop, attendees were asked to declare which of the following four descriptions of biorelevant dissolution testing they most agree with by using a measurement scale of 1-4 ( 1 = most favorable, 4 = least favorable):

Description 1: Differentiates between bio-in-equivalent products

Description 2: Reflects intraluminal conditions (e.g., intraluminal composition and hydrodynamics)

Description 3: Mimics in vivo dissolution

Description 4: Differentiates between products that are not identically manufactured

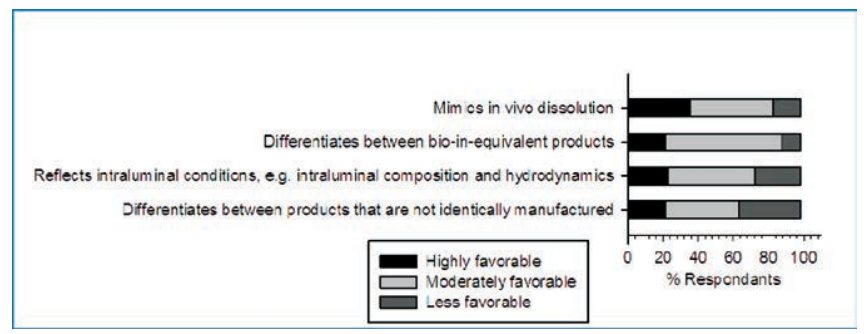

Figure 1. Total scores of various suggested descriptions of biorelevant dissolution testing.

Based on Figure 1, the description "mimics in vivo dissolution" received the most favorable score (i.e., received a majority of votes for the best description of the phrase "biorelevant dissolution testing"). In addition, at the end of the workshop at least $2 / 3$ of the attendees agreed that dissolution testing does not differentiate between bioequivalent and non-bioequivalent products and that dissolution testing is typically not clinically relevant.

BCS media are biorelevant for BCS Class 1 drugs in IR products. Although IVIVCs are generally expected for ER products, IVIVC methods for IR products are not well developed and readily available. In practice, biorelevant dissolution testing is not easy to perform, and the selection of dissolution media is not straightforward.
The FIP Dissolution/Drug Release Focus Group will publish a detailed summary report of the discussions at both the 2012 AAPS symposium and 2013 AAPS workshop in the near future. The FIP FG plans to draft a position paper on the topic following an additional 2014 workshop planned in Europe.

\section{ABBREVIATIONS}

API active pharmaceutical ingredient

BCS Biopharmaceutics Classification System

BE bioequivalence

BfArM German Federal Institute for Drugs and Medical Devices

EMA European Medicines Agency

ER extended release

FaSSiF fasted-state intestinal fluid

FeSSiF fed-state intestinal fluid

FDA Food and Drug Administration

FG focus group

FIP Fédération International Pharmaceutique (Intl. Pharm. Federation)

Gl gastrointestinal

$\mathrm{ICH} \quad$ International Conference on Harmonization of Technical Requirements for Registration of Pharmaceuticals for Human Use

IMI Innovative Medicines Initiative

IR immediate release

IVIVC in vitro-in vivo correlation

MRT mean residence time

OGD Office of Generic Drugs

ONDQA Office of New Drug Quality Assessment

OrBiTo oral biopharmaceutics tools

PBPK physiologically based pharmacokinetic modeling

QbD quality by design

QC quality control

SDS sodium dodecyl sulfate

The FIP Focus Group on Dissolution and Drug Release planned this AAPS Workshop on Biorelevant In Vitro Performance Testing of Orally Administered Dosage Forms. 


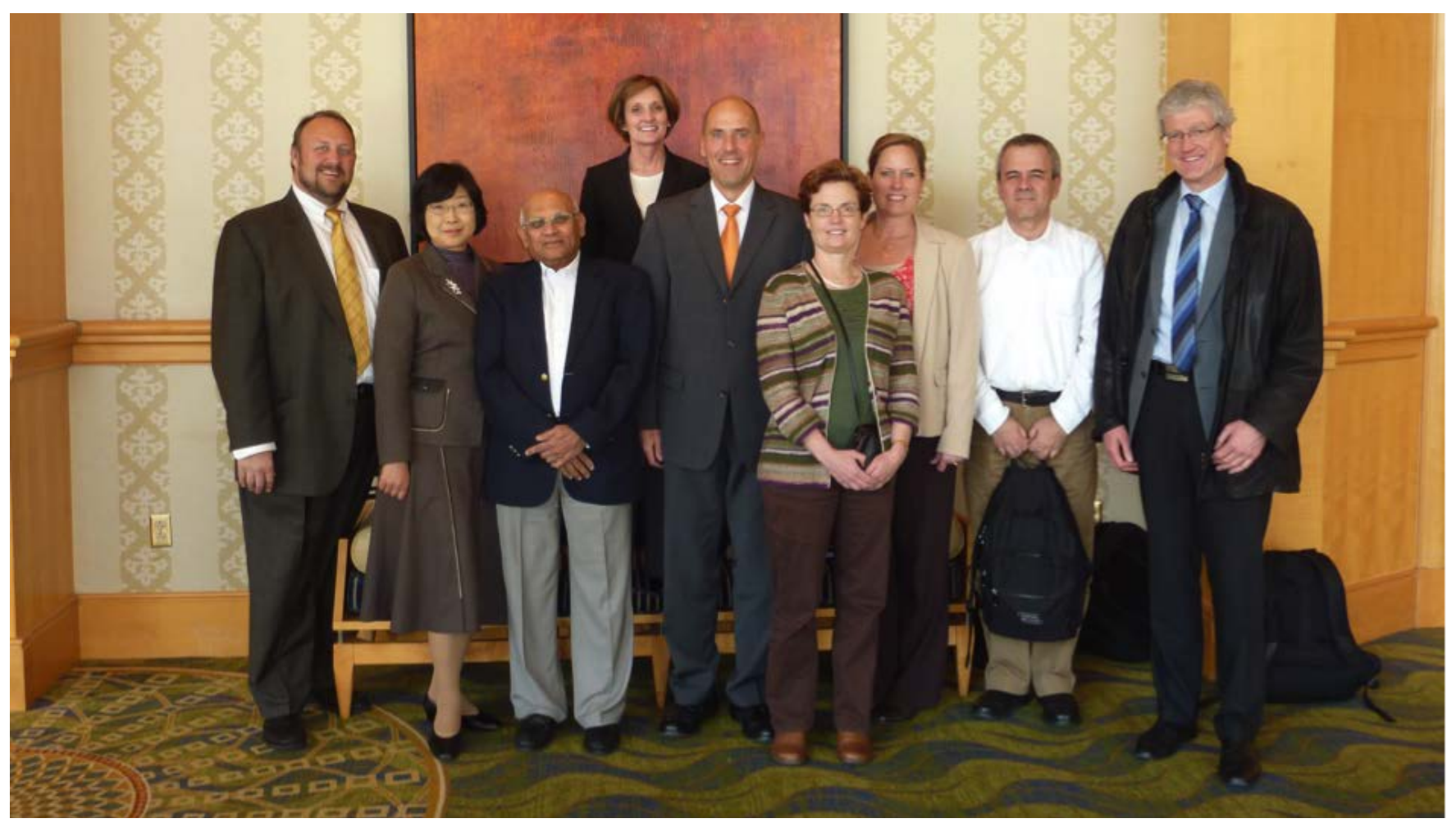

Programming committee from left to right: Todd L. Cecil, Chikako Yomota, Vinod P. Shah, Cynthia K. Brown, Johannes Kraemer, Lucinda Buhse, Amy R. Barker, Christos Reppas, Horst-Dieter Friedel (missing: Susanne Keitel, Michael Morris).

\section{PROGRAMMING COMMITTEE}

Cynthia K. Brown, Eli Lilly and Company, cochair

Christos Reppas, Ph.D., National and Kapodistrian University of Athens, cochair

Amy R. Barker, Ph.D., Eli Lilly and Company

Lucinda Buhse, Ph.D., U.S. Food and Drug Administration

Todd L. Cecil, Ph.D., U.S. Pharmacopeial Convention

Horst-Dieter Friedel, Ph.D., Bayer Pharma Aktiengesellschaft

Susanne Keitel, Ph.D., European Directorate for the Quality of Medicines and HealthCare

Johannes Kraemer, Ph.D., PHAST

Michael Morris, Ph.D., Irish Medicines Board
Vinod P. Shah, Ph.D., International Pharmaceutical Federation (FIP)

Chikako Yomota, Ph.D., National Institute of Health Science, Japan

\section{REFERENCES}

1. Dressman, J. B.; Amidon, G. L.; Reppas, C.; Shah, V. P. Dissolution testing as a prognostic tool for oral drug absorption: immediate release dosage forms. Pharm. Res. 1988, 15 (1), 11-22.

2. Butler, J. M.; Dressman, J. B. The developability classification system: application of biopharmaceutics concepts to formulation development. J. Pharm. Sci. 2010, 99 (12), 4940-4954. 